\title{
September 2016 Critical Care Case of the Month
}

\author{
Clement $U$. Singarajah, MD \\ Samir Sultan, DO \\ Phoenix VA Medical Center \\ Phoenix, AZ USA
}

\section{Clinical History}

A 66-year-old man was admitted to the ICU in complete heart block with borderline hypotension. After cardiology consultation, a decision was made to place an urgent transvenous pacer. The transvenous pacer was place without use fluoroscopy from an right internal jugular venous (IJV) approach using real time ultrasound by two very experienced operators. The ultrasound confirmed right IJV placement and the pacer was found to capture and pace appropriately without any complications. A post placement CXR was obtained (Figure 1).

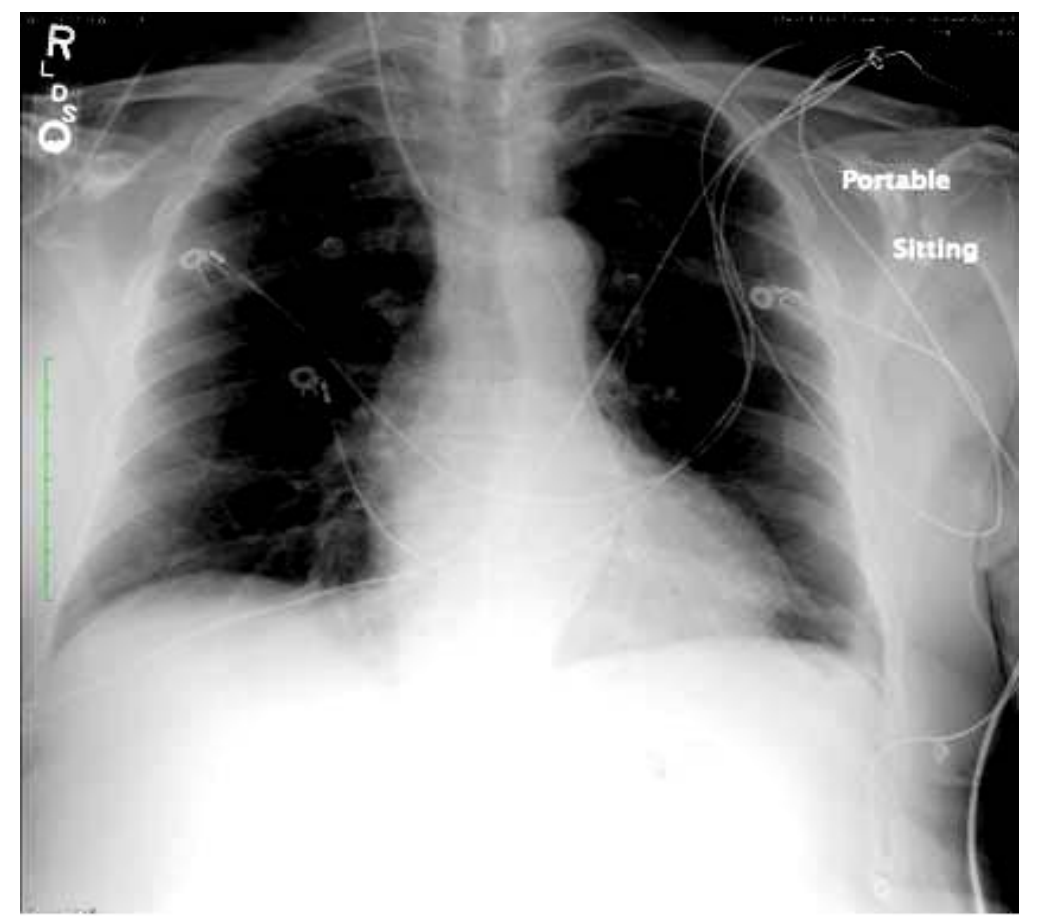

Figure 1. Portable chest x-ray after RIJV transvenous pacer (TVP).

What does the chest $x$-ray show?

1. A persistent left SVC

2. Normal placement of the RIJV TVP

3. Right pneumothorax

4. TVP pacer passing from RIJV and then into the aorta and left ventricle

5. TVP pacer passing outside heart and pacing epicardially 


\section{Correct! \\ 2. Normal placement of the RIJVP TVP}

There is no pneumothorax. The tip of the pacer appears to be in the appropriate position however, the pacer wire crosses from the right to the left side. Although the pacer appears to be working correctly, this chest x-ray appearance could suggest several possibilities.

Based on the chest x-ray, what would you do next?

1. Ensure that the device is not in the arterial circulation

2. Remove the device and try to place again.

3. Nothing, the device is safe to use as a TVP

4. 1 and 3

5. All of the above are acceptable 


\section{Correct!}

\section{1 and 3}

The device appears to be functioning correctly but the chest x-ray appearance is concerning for possible arterial placement. To ensure that the device is not in the arterial circulation, aspirate blood from the device and obtain a blood gas from this sample and a simultaneous ABG. Another method is to connect a pressure transducer to check for arterial or venous waveform. These can be done quickly at the bedside and save an unnecessary escalation of care. If these tests suggest arterial cannulation if arterial is still suspected, the device should $\underline{\text { NOT }}$ be removed as brisk bleeding could occur into the mediastinum.

A pressure tracing was consistent with venous pressure and blood aspirated was consistent with a venous sample. A thoracic CT scan was ordered because of the unusual appearance of the pacer (Figure 2).
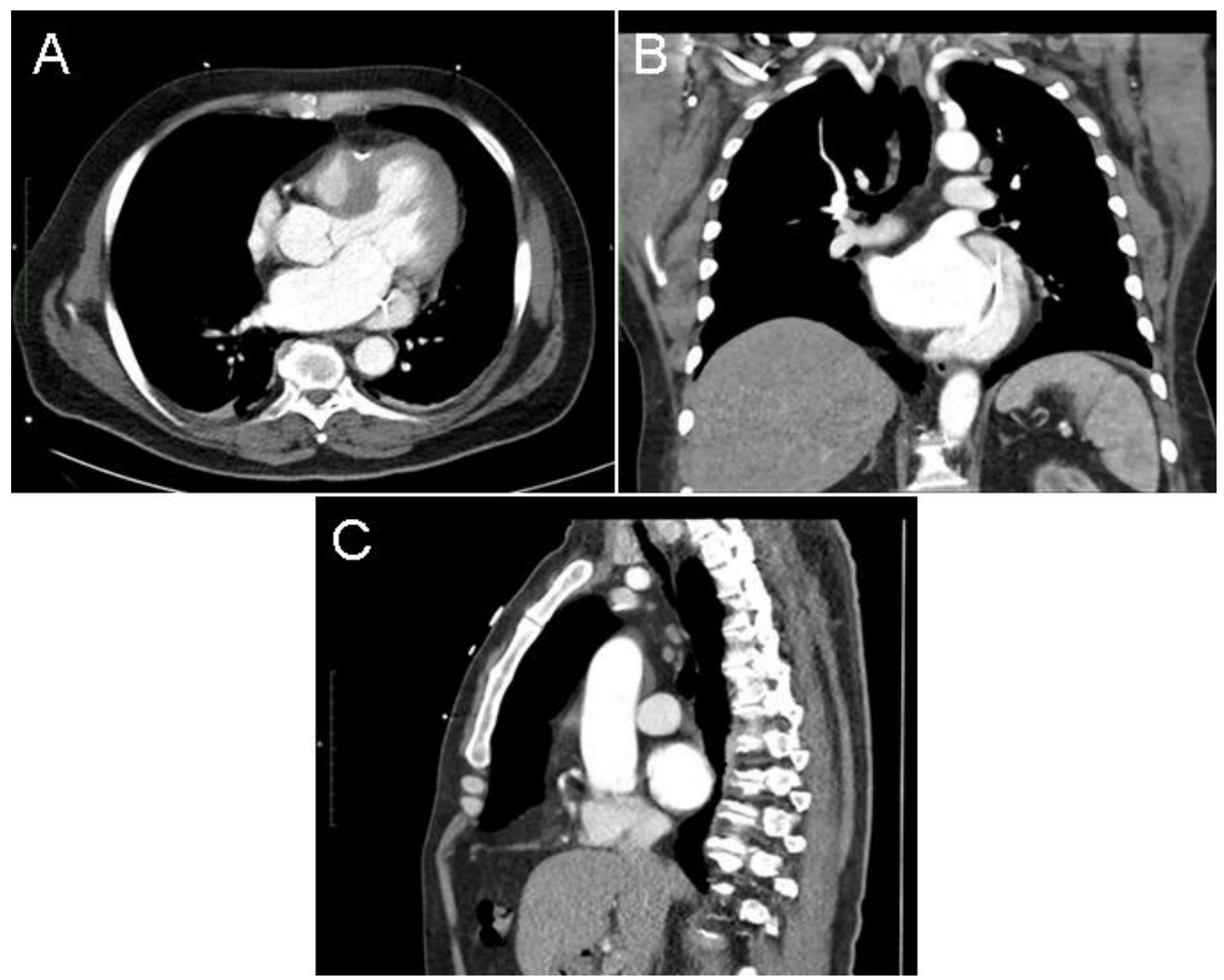

Figure 2. Representative images from thoracic CT scan in soft tissue windows in axial $(A)$, coronal (B) and sagittal (C) views. 
Which of the following are false about the condition?

1. A normal right SVC is not commonly found in this anomaly

2. Most patients present because they are symptomatic from a right left intracardiac shunt

3. The anomalous drainage is most commonly into the coronary sinus

4. This is the most common congenital abnormality of the venous system in the chest

5. All are false 


\section{Correct! \\ 2. Most patients present because they are symptomatic from a right left intra- cardiac shunt}

The patient has a persistent left superior vena cava with anomolous drainage of the right internal jugular vein to the coronary sinus. This is the most common congenital abnormality of the venous system in the chest (1-4). Most patients are asymptomatic. The tip of the pacer appears to perforate the right ventricle (Figure 3). However, the lack of a pericardial effusion suggests that it is probably just tenting the muscle up. (Gotway MB personal communication).

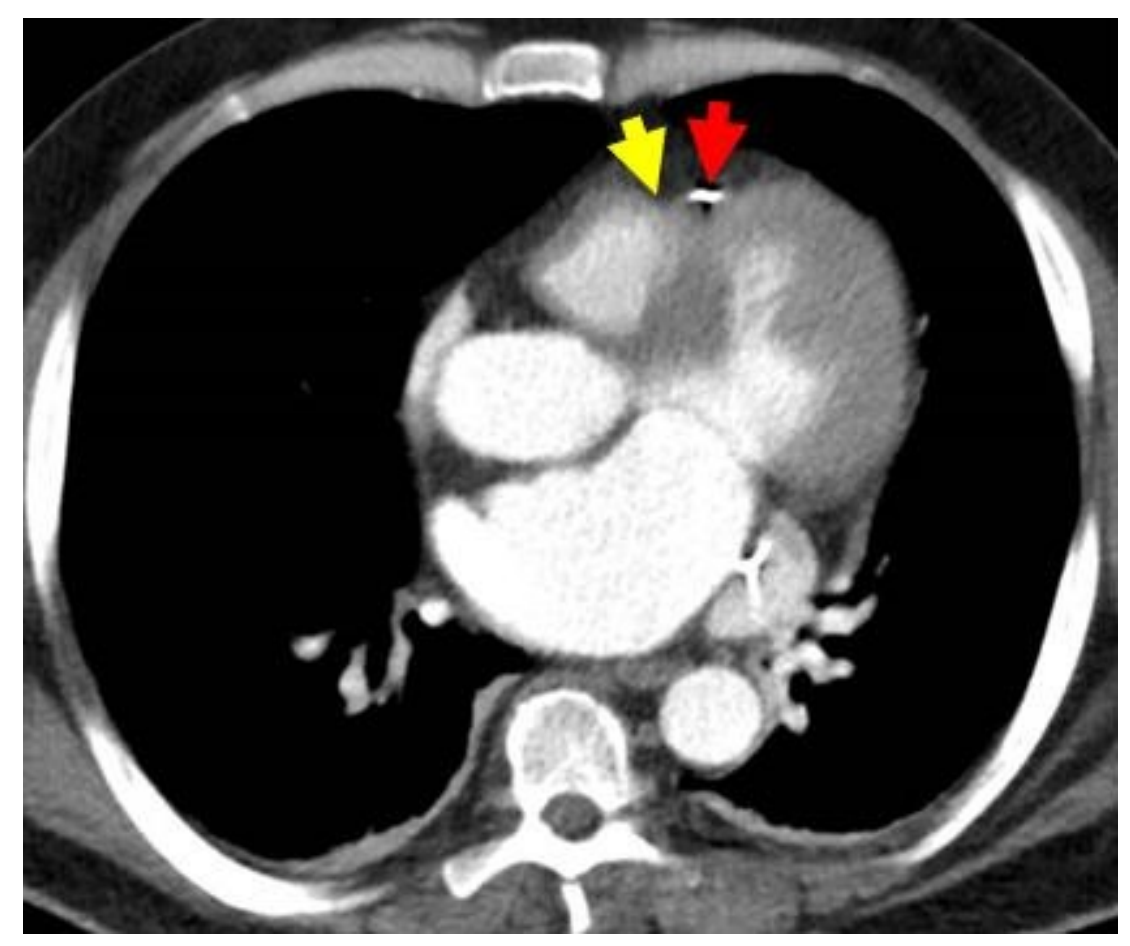

Figure 3. The pacer tip (red arrowhead) is upturned and projects beyond the RV musculature (at tip of yellow arrowhead) probably tenting the muscle up.

What should be done at this point?

1. Echocardiogram for associated conditions such as VSD, single atrium, pulmonary stenosis and ASD.

2. No further evaluation is needed.

3. Remove the pacer wire.

4. 1 and 3.

5. None of the above. 


\section{Correct! \\ 1. Echocardiogram for associated conditions such as VSD, single atrium, pulmonary stenosis and ASD.}

The patient should be evaluated for the other congenital abnormalities which are associated with the anomalous drainage of the internal jugular vein (4). Echocardiogram revealed no such abnormalities and the pacer continued to function normally until a permanent pacemaker was inserted.

\section{References}

1. Pretorius PM, Gleeson FV. Case 74: right-sided superior vena cava draining into left atrium in a patient with persistent left-sided superior vena cava. Radiology. 2004;232 (3): 730-4. [CrossRef] [PubMed]

2. Smyth YM, Barrett CD, Fahy GJ. Images in cardiology. Biventricular pacemaker implant in a patient with persistent left sided superior vena cava. Heart. 2005;91 (11): 1427. [CrossRef] [PubMed]

3. Rose M, Gross L, Protos A. Transvenous pacemaker implantation by way of an anomalous left superior vena cava. J Thorac Cardiovasc Surg. 1971;62:965-6. [PubMed]

4. Povoski SP, Khabiri H. Persistent left superior vena cava: Review of the literature, clinical implications, and relevance of alterations in thoracic central venous anatomy as pertaining to the general principles of central venous access device placement and venography in cancer patients World J Surg Oncol. 2011; 9: 173. [CrossRef] [PubMed] 